\title{
A case of Brugada syndrome patient undertaken total intravenous anesthesia with remifentanil
}

\author{
Su-Yeon Lee ${ }^{1}$, Chunghyun Park ${ }^{2}$, Min-Young Kim², Seomin Park ${ }^{2}$, and Yun Sic Bang ${ }^{2}$ \\ Department of Anesthesiology and Pain Medicine, ${ }^{1} \mathrm{CHA}$ University Hospital, Seoul, ${ }^{2} \mathrm{CHA}$ Bundang Medical Center, CHA \\ University, Seongnam, Korea
}

Brugada syndrome (BS) is an idiopathic ventricular fibrillation characterized by a right bundle branch block, and ST segment elevation in the right precordial leads (V1 to V3) without evidence of underlying structural heart disease and susceptibility to ventricular arrhythmias that may culminate in syncope or sudden death [1]. Although diagnosis of BS before the operation and anesthesia is very critical, it is well recognized that the classical electrocardiography (ECG) pattern can be intermittent and is often concealed, and there are no family history, no history of unexplainable syncope, tachycardia, no typical ECG findings in $50 \%$ patients, making the diagnosis not always apparent [2]. We describe a case of total intravenous anesthesia (TIVA) with propofol and remifentanil in a female patient with BS.

A 56-year-old woman was presented for perforated appendicitis with periappendicial abscess. She had no known underlying disease and no past history. Preoperative ECG revealed right bundle branch block and ST segment elevation in the precordial lead (V1-3) (type I ECG abnormality), which suggested BS (Fig. 1B). She had no past history of syncope, tachycardia or chest pain, except family history of nocturnal sudden death of her brother ten years ago. A previous ECG from 5 years ago showed incomplete right bundle branch block (I-RBBB) (Fig. 1A).

Having been informed of the associated risks, the patient was taken to the operating room and routine monitors were applied. A radial arterial pressure was monitored. Prior to the induction of anesthesia, automatic defibrillator pads (Physio-Control Quick-Combo Electrodes, Medtronic, USA) for cardioversion were attached to her anterior chest. ECG monitoring from external defibrillator was done simultaneously. The patient's blood pressure (BP), heart rate (HR), oxygen saturation was 132/78
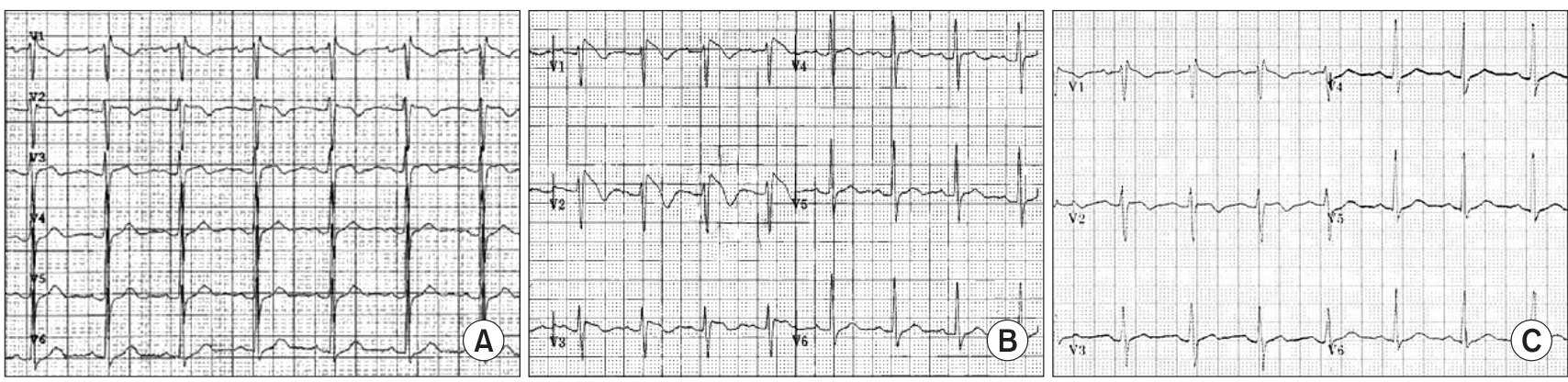

Fig. 1. (A) ECG findings from 5 years ago shows incomplete right bundle branch block, (B) preoperative ECG shows Brugada syndrome displaying coved-type ST-segment elevation $\geq 2 \mathrm{~mm}$ in more than one right precordial lead (V1-V3), followed by negative T waves, (C) immediate postoperative ECG at intensive care unit shows incomplete right bundle branch block.

Corresponding author: Yun Sic Bang, M.D., Department of Anesthesiology and Pain Medicine, CHA Bundang Medical Center, CHA University, 351, Yatap-dong, Bundang-gu, Seongnam 463-712, Korea. Tel: 82-31-780-5431, Fax: 82-31-701-9433, E-mail: pocut@hanmail.net

(c) This is an open-access article distributed under the terms of the Creative Commons Attribution Non-Commercial License (http:// creativecommons.org/licenses/by-nc/3.0/), which permits unrestricted non-commercial use, distribution, and reproduction in any medium, provided the original work is properly cited. 
mmHg, 92 beats/min and 99\%, respectively. Anesthesia was induced with intravenous target controlled infusion (TCI) of propofol with the effect site concentration (Cet) of $3.5 \mu \mathrm{g} / \mathrm{ml}$ and remifentanil with Cet of $3 \mathrm{ng} / \mathrm{ml}$, using TCI pump. Verbal response was lost when the propofol reached the Cet of $0.8 \mu \mathrm{g} /$ $\mathrm{ml}$ and remifentanil at $2 \mathrm{ng} / \mathrm{ml}$. The bispectral index at this moment was 60 and $0.6 \mathrm{mg} / \mathrm{kg}$ of rocuronium was administered. Intubation was performed when the Cet of propofol reached $3.5 \mu \mathrm{g} /$ and remifentanil $2.5 \mathrm{ng} / \mathrm{ml}$. The patient's BP and HR recorded 121/68 $\mathrm{mmHg}$, and 82 beats/min after intubation. Laparoscopic appendectomy lasted 40 minutes. We did not administer anticholinesterase to antagonize the rocuronium. The patient recovered spontaneously and was extubated when propofol reached Cet of $0.5 \mu \mathrm{g} / \mathrm{ml}$ and remifentanil $1.0 \mathrm{ng} / \mathrm{ml}$. The BP and HR was 140/68 mmHg, and 85 beats/min when the patient was extubated. The patient was transferred to the intensive care unit with the automatic defibrillator pads. Twelve leads ECG on the arrival of intensive care unit showed normal, except I-RBBB (Fig. 1C). The vital signs were stable and postoperative pain was also controlled with intravenous patient controlled analgesics. The cardiac rhythm was uneventful during surgery and at intensive care unit. The patient was transferred to the general ward 24 hours after the operation, and there was no episode of tachyarrhythmia throughout her hospital stay.

A history of unexplained syncope or rapid palpitations with presyncope is concerning. Fifty percent of diagnosis of BS is made with family history and typical ECG findings [2]. In this case, despite having family history, the patient did not know about her disease.

In a review of 163 cases, males $(n=150)$ far outnumbered females ( $n=13)$ and $58 \%$ of patients were of Asian ancestry. The number of male patients with BS is 11 times more than that of female [3]. This is the first female case report in Korea, which is related to anesthesia.

Because ECG can be normalized transiently in up to $50 \%$ of cases, the intermittent nature of these findings can make diagnosis of the BS difficult. In this case, ECG findings from 5 years ago, in intensive care unit, and 24 hours Holter test showed only I-RBBB pattern. I-RBBB is a common ECG finding on preoperative screening, and is not considered seriously in most cases. It is not possible to undergo pharmacological drug challenge and electro-physiologic stimulation for all the patients who have I-RBBB findings, but we have to make an effort to take careful history of patients and their family to find out BS. More recently, it has been recommended that all patients with isolated type I ECG abnormality should be considered at a risk of sudden death. It would seem sensible to treat these patients undergoing anesthesia as having the condition [2]. In general, implantable cardioverter-defibrillator implantation is recommended for all patients who have already had symptoms and for asymptomatic patients in whom the electro-physiologic stimulation induces ventricular arrhythmias or those with family history, especially if they present a spontaneous type I ECG pattern [2]. The risk of ventricular arrhythmias in the perioperative period is of concern as the pharmacological and physiological changes that occur during anesthesia are implicated in precipitating such events. An understanding of modulating agents and conditions is essential for planning of appropriate techniques and prevention of crisis. Depth of anesthesia should be balanced to minimize these effects, as bradycardia or increased vagal tone as a result of surgical stimulation have also been implicated in the development of Brugada ECG changes [4]. We used propofol-remifentanil for induction and maintenance of anesthesia because major advantages of TIVA are hemodynamic stability and reduced hemodynamic response during the emergence when compared with balanced anesthesia. Although, the most common opioid used in BS is fentanyl [4], Ahn and Kim [5] reported successful case of using remifentail. In our case, we successfully managed intubation, maintenance, and fully awake extubation without hemodynamic instabilities or tarchyarrhythmic events using TIVA. The range of blood pressure and heart rate changes were within 9 and $11 \%$, respectively, of the preoperative value.

In conclusion, this case shows that I-RBBB can be the only sign of BS, and we have to take a careful history of patients with I-RBBB, both male and female. TIVA is considered as one of the available anesthetic methods for BS patients.

\section{References}

1. Brugada P, Brugada J. Right bundle branch block, persistent ST segment elevation and sudden cardiac death: a distinct clinical and electrocardiographic syndrome. A multicenter report. J Am Coll Cardiol 1992; 20: 1391-6.

2. Brugada R, Brugada J, Antzelevitch C, Kirsch GE, Potenza D, Towbin JA, et al. Sodium channel blockers identify risk for sudden death in patients with ST-segment elevation and right bundle branch block but structurally normal hearts. Circulation 2000; 101: 510-5.

3. Alings M, Wilde A. "Brugada" syndrome: clinical data and suggested pathophysiological mechanism. Circulation 1999; 99: 666-73.

4. Dulu A, Pastores SM, McAleer E, Voigt L, Halpern NA. Brugada electrocardiographic pattern in a postoperative patient. Crit Care Med 2005; 33: 1634-7.

5. Ahn YH, Kim JY. Anesthetic management of a patient with Brugada syndrome for one-lung ventilation by total intravenous anesthesia with propofol and remifentanil: A case report. Anesth Pain Med 2009; 4: 310-3. 